\title{
IMPLANTAÇÃO DA VIGILÂNCIA SANITÁRIA EM CAMPOS SALES-CE
}

\section{CORTEZ, Daniela X.*; CORTEZ, Francisca O. X.; LEITE, Renata M.; MOREIRA, Maria R.}

\author{
Faculdade Leão Sampaio - Juazeiro do Norte (CE), Brasil. \\ Universidade Regional do Cariri - Crato (CE), Brasil.
}

Recebido em: 14/01/2015; Aceito: 22/04/2015; Publicado: 25/08/2015

\section{RESUMO}

A Vigilância Sanitária (VISA) como uma das partes integrantes do Sistema Único de Saúde enfrenta vários desafios que não se restringem apenas às discórdias entre questões mercantis versus saúde, mas às várias demandas externas como a qualidade da formação profissional, a produção e disseminação de conhecimentos e informações atualizadas, a pontos conceituais e doutrinários que envolvem todo o arcabouço da área da saúde, e até mesmo as tecnologias que envolvem todo o meio em que se insere a vigilância sanitária. O presente estudo teve como objetivo conhecer e analisar o processo de implantação da Vigilância Sanitária no município de Campos Sales-CE. Já do ponto de vista metodológico trata-se de um estudo de caráter exploratório descritivo com abordagem qualitativa. A coleta dos dados se deu através da entrevista parcialmente estruturada e transcrita fidedignamente, a fim de atingir os objetivos propostos. Conclui-se que as vantagens da implantação desse setor são inúmeras, uma vez que se trata de uma ferramenta tão importante para saúde e que a única desvantagem é a falta de compromisso por parte dos comerciantes que não seguem as leis atribuídas pelo órgão, dificultando assim o trabalho dos funcionários da VISA, que precisam fiscalizar tudo isso, além das outras atividades em outros setores que os mesmos executam.

Palavras - chave: Vigilância Sanitária, Implantação, Usuários

\begin{abstract}
The CDC as one of the integral parts of the National Health System faces several challenges that are not restricted to the discord between market issues versus health, but several external demands as the quality of vocational training, production and dissemination of knowledge and updated information the conceptual and doctrinal points that involve the entire health care framework, and even the technologies that involve the whole environment in which it operates health surveillance . This study aimed to investigate and analyze the implementation process of the Health Surveillance in the city of Sales- EC Campos. Have a methodological point of view it is a descriptive exploratory qualitative study, data collection was made through the partially structured interviews and transcribed faithfully in order to achieve the proposed objectives. It is concluded that the implementation of this sector advantages are numerous, since it is such an important tool for health and that the only disadvantage of this is the lack of commitment on the part of traders that do not follow the laws given by the body, making it difficult so the work of VISA employees, who need to supervise all this, in addition to other activities in other sectors that they perform
\end{abstract}

Keywords: Health Monitoring, Implementation, Use

*Daniela Cortez - Faculdade Leão Sampaio

Rua Coronel Baleco, 404, Centro - Campos Sales, Ceará, CEP: 63150000. e-mail: daniela.cortez@hotmail.com 


\section{INTRODUÇÃO}

Em 1990, com uma regulamentação, através da Lei Orgânica da Saúde, foi instituído um novo modelo de assistência à saúde dos cidadãos brasileiros, conhecido como o Sistema Único de Saúde (SUS), no qual suas ações devem estar norteadas a partir dos seguintes princípios: universalidade de cobertura e eqüidade; descentralização dos serviços para os estados e municípios, que participarão do financiamento; não concorrência nas unidades e hierarquização entre as ações de saúde da União, dos estados e dos municípios; participação complementar do setor privado na oferta de serviços; prioridade para as atividades preventivas; e controle social através dos Conselhos de Saúde e participação da comunidade. O SUS tornou-se, assim, o sistema integrado de assistência à saúde, garantido pelo Estado brasileiro e de responsabilidade administrativa do Ministério da Saúde (PEREIRA, 1995). A referida Lei afirma, em seu artigo $6^{\circ}$, que integram o campo de atuação do SUS, a vigilância epidemiológica, a Vigilância Sanitária, a saúde do trabalhador e a assistência terapêutica integral, incluindo a assistência farmacêutica (LUCCHESE, 2001).

Devido às mudanças na política social do país, especialmente na área da saúde, a partir de 1990, surgiam desafios quanto à forma de planejar, gerir e avaliar as políticas de saúde em contextos descentralizados e autônomos, os quais exigem articulação intersetorial e intergovernamental e o incremento da participação da comunidade nas decisões do setor (PIOVESAN et al., 2005).

A Lei n. ${ }^{\circ}$ 9.782, proclamada pelo Congresso Nacional em 27 de janeiro de 1999, estabelece as competências exclusivas da Agência Nacional de Vigilância Sanitária (ANVISA) e as partilhadas pelos outros níveis de gestão e afirma no artigo $6 .^{\circ}$ que:
A Agência terá por finalidade institucional promover a proteção da saúde da população, por intermédio do controle sanitário da produção e da comercialização de produtos e serviços submetidos à vigilância sanitária, inclusive dos ambientes, dos processos, dos insumos e das tecnologias a eles relacionados, bem como o controle dos portos, aeroportos e fronteiras (COSTA, 2001).

$\mathrm{Na}$ perspectiva de superar algumas dificuldades apontadas pelo SUS, os gestores assumiram o compromisso público da construção do PACTO PELA SAÚDE 2006, com base nos princípios constitucionais do SUS, ênfase nas necessidades de saúde da população e que implicou o exercício simultâneo de definição de prioridades articuladas e integradas nos três componentes: Pacto pela Vida, Pacto em Defesa do SUS e Pacto de Gestão do SUS. Onde o Pacto de Gestão estabeleceu as diretrizes para o financiamento do sistema e respectivos blocos, criando, dentre eles, o bloco de Vigilância em Saúde, composto pelos componentes: I. Vigilância epidemiológica; II. Promoção da saúde; III. Vigilância da situação de saúde; IV. Vigilância em saúde ambiental; V. Vigilância da saúde do trabalhador; VI. Vigilância Sanitária (DE SETA; REIS; DELAMARQUE, 2010).

Para Costa et al. (2002), a Vigilância Sanitária representa um conjunto de ações capazes de eliminar, diminuir ou prevenir riscos à saúde e de intervir nos problemas sanitários decorrentes do meio ambiente, da produção e da circulação de bens e da prestação de serviços de interesse da saúde, envolvendo: o controle de bens de consumo que, direta ou indiretamente, se relacionem com a saúde, compreendidas todas as etapas de processo, da produção ao consumo; o controle da prestação de serviços que se relacionam direta ou indiretamente com a saúde. 
Acredita-se que, para a descentralização das ações de VISA resultar em maior impacto para a proteção da saúde da população, as responsabilidades entre os gestores do SUS devem ser pactuadas e implementadas de forma integrada entre os entes federativos. Se tal processo é ainda hoje marcado, por um lado, por incertezas quanto a seu alcance e implicações, ele tem, por outro lado, se revelado um campo fértil de experiências municipais inovadoras, seja em relação ao incremento da oferta de serviços, à reorganização do modelo assistencial, ou ainda à revisão das formas de relação público-privado, entre outros aspectos (COHEN; MOURA, TOMAZELLI, 2004).

Diante da importância da Vigilância Sanitária para a saúde da população e como parte integrante da Saúde, percebe-se que sua implantação ainda precisa ser compreendida e explicada, uma vez que sua municipalização não se constitui um movimento discreto e sim um processo complexo estabelecido pelas determinações políticas, sociais e econômicas da realidade brasileira.

A fim de responder a tais questionamentos, o presente estudo tem por objetivo: conhecer e analisar a implantação da Vigilância Sanitária no município de Campos Sales, CE, favorecendo o desenvolvimento de novas perspectivas para a técnica em questão, buscando uma mudança no contexto local com vistas à construção de um sistema de Vigilância Sanitária voltado para a promoção da qualidade de vida da população e do conseqüente exercício da cidadania

\section{METODOLOGIA}

\subsection{TIPO DE PESQUISA}

O estudo apresenta caráter exploratório descritivo que, "Pretende descrever com exatidão os fatos e fenômenos de determinada realidade, o que exige do pesquisador uma série de informações sobre o que se deseja pesquisar, como por exemplo, a população, a amostra, os objetos e as questões de pesquisa.” (TRIVINOS 1987, p.110).

De natureza qualitativa, que "Por meio do método qualitativo, o investigador entra em contato direto e prolongado com o indivíduo ou grupos humanos, com o ambiente e a situação que está sendo investigada, permitindo um contato de perto com os informantes" (LAKATOS; MARCONI, 2004, p.267). A metodologia qualitativa preocupase em analisar e interpretar aspectos mais importantes, descrevendo o comportamento humano em sua complexidade. Proporciona uma análise minuciosa sobre as investigações, os costumes, as atitudes, as tendências de comportamento, etc (LAKATOS; MARCONI, 2004).

\subsection{LOCAL DA PESQUISA}

O estudo será realizado no Setor de Vigilância Sanitária do município de Campos Sales, CE. Localizada na microrregião da Chapada do Araripe, mesorregião do Sul Cearense. Com população de 26.506 habitantes.

\subsection{PERÍODO DA PESQUISA}

O início da elaboração do trabalho do projeto ocorreu no mês de Fevereiro de 2013. A coleta de dados foi desenvolvida em Fevereiro de 2013, seguindo as etapas de: elaboração, desenvolvimento, avaliação e finalização.

\subsection{POPULAÇÃO E AMOSTRA}

Profissionais que participaram ou conhecem a história da implantação da Vigilância Sanitária no município de Campos Sales-CE, e que 
atuam no setor referido. A amostra foi constituída pelos profissionais que aceitaram participar do estudo assinando termo de consentimento livre e esclarecido.

\subsection{INSTRUMENTO E COLETA DE DADOS}

O instrumento utilizado foi a entrevista parcialmente estruturada e transcrita fidedignamente, a fim de atingir os objetivos propostos e coerentes com o tipo de estudo. "A entrevista constitui um instrumento eficaz na recolha de dados fidedignos para a elaboração de uma pesquisa, desde que seja bem elaborada, bem realizada e interpretada" (ANDRADE, 2003). Segundo Gil (2002), a entrevista é parcialmente estruturada "quando é guiada por relação de pontos de interesse que o entrevistador vai explorando ao longo de seu curso".

\subsection{APRESENTAÇÃO DOS DADOS}

Os dados coletados foram categorizados e receberam tratamento por meio da análise de conteúdo.

\subsection{ASPECTOS ÉTICOS E LEGAIS DA PESQUISA}

O estudo foi desenvolvido considerando-se os princípios éticos da Resolução n¹96 de 10 de outubro de 1996, do Conselho Nacional de Saúde, que normatiza pesquisas envolvendo seres humanos. Atendendo a esta resolução, os sujeitos receberam previamente à coleta de dados, um Termo de Consentimento Livre e Esclarecido (TCLE) que, após leitura e aceite, foi assinado pelos participantes para prosseguimento com a coleta. O TCLE descreve os objetivos do estudo, a técnica de coleta de dados, a garantia de anonimato dos sujeitos, do sigilo e do compromisso de que as informações gravadas foram destinadas exclusivamente a fins técnicos e científicos.

\section{RESULTADOS E DISCUSSÃO}

A pesquisa foi realizada no Setor de Vigilância Sanitária no município de Campos Sales, Estado do Ceará, que presta assistência de fiscalização e prevenção dos riscos a saúde dos moradores da cidade em estudo.

Foi realizado um questionário com perguntas simples e objetivas, contendo questões importantes para conhecer e analisar o processo de implantação da Vigilância Sanitária segundo os profissionais, que exercem suas atividades na VISA. Os questionários foram aplicados por uma estudante do curso de pós-graduação em Gestão em Saúde.

Descreve-se inicialmente a caracterização da amostra desta pesquisa de forma que se possa identificar o conhecimento dos profissionais sobre a VISA, como se implantou a Vigilância Sanitária em Campos Sales, o processo de implantação da VISA e vantagens e desvantagens de implantação do Setor de Vigilância Sanitária.

Cabe ressaltar que os resultados obtidos no presente trabalho foram através do questionário respondido por 06 profissionais da Vigilância Sanitária da cidade em questão, onde os mesmos foram selecionados de forma aleatória. 
Tabela 1. Distribuição do sexo, faixa etária, escolaridade e cargo relatado pelos profissionais que responderam ao questionário aplicado na VISA.

\begin{tabular}{|c|c|c|c|c|}
\hline PSEUDÔNIMO & SEXO & IDADE & ESCOLARIDADE & CARGO \\
\hline \multirow{2}{*}{ Acácia Branca } & Masculino & 19 anos & Nível médio & Inspetor sanitário \\
\hline Camélia Branca & Feminino & 58 anos & Nível superior & Chefe de Insp. \\
\hline Hortência & Masculino & 20 anos & Nível médio & Inspetor sanitário \\
\hline Jasmim & Masculino & 24 anos & Nível superior & Inspetor sanitário \\
\hline Marquídea & Masculino & 30 anos & N. superior incomp & Inspetor sanitário \\
\hline
\end{tabular}

\subsection{CATEGORIZAÇÃO TEMÁTICA DAS FALAS}

\subsubsection{Categoria I: Conhecimento dos profissionais sobre a VISA}

Conhecimento é o ato de abstrairmos idéia de alguma coisa ou acontecimento. O estudo do conhecimento é denominado gnosiologia. Existem vários conceitos de conhecimento podendo ser simplesmente definido como aquilo que se sabe de algo ou de alguém lembrando que ao abordarmos o tema conhecimento haverá sempre a necessidade de fornecimento de dados (HESSEN, p.71. 2000).

O campo de conhecimentos de qualquer área vai sendo lentamente construído e essa construção reflete no amadurecimento dos profissionais, baseado na prática, na intuição e nos saberes incorporados de outras disciplinas. A construção do corpo de conhecimentos depende da reunião e da organização dos resultados obtidos através de investigações, observações e achados. Esse é o papel da teoria; isto é, os conhecimentos parciais são reunidos pela criação teórica.

O conhecimento pode ainda ser definido como o produto do processo de teorias e conceitos. Dentre as várias categorias de conhecimentos destaca-se o conhecimento científico, que presa pela aprovação e constatação no intuito de explicar de modo racional aquilo que se observa.

Esta categoria, analisada a partir de depoimentos dos participantes da pesquisa, retratando o conhecimento sobre a VISA. Os seguintes enunciados revelam essa realidade:

"A Vigilancia Sanitária
tem como dever
fiscalizar e prevenir
possíveis riscos a


saúde, qualquer tipo de serviço que preste atendimento a saúde dever ser fiscalizado pela VISA, desde os comércios, hospitais e clínicas até restaurantes, hotéis $e$ postos de gasolina". (Acácia Branca)

“A VISA é um órgão que tem como principal finalidade impedir que a saúde humana seja exposta a riscos, combatendo as causas dos efeitos nocivos que lhes forem gerados". (Camélia Branca)

“A Vigilância sanitária é uma forma de prevenção de riscos a saúde da população". (Hortência)

Esta categoria revela que os profissionais possuem conhecimentos sobre o que significa a Vigilância Sanitária, sua extensa função e percebem sua importância na cidade.

A noção de vigilância, segundo Sato (1996), tem pelo menos duas posições, a primeira que, através do conceito de Vigilância à Saúde, busca sinalizar a ampliação das responsabilidades dos serviços de saúde, incluindo aí não apenas o atendimento aos danos e ao controle de certos riscos, mas também o controle das condições de vida e de determinantes ambientais do processo saúde-doença e a segunda que, embora assumindo a necessidade de ampliação do raio de ação dos sistemas de vigilância epidemiológica, busca preservar certas características específicas desse conjunto de atividades.

O assunto é amplo, porém pouco explorado nas instituições de ensino que estão mais preocupadas em formar profissional deixando de lado o mais importante a ser trabalhado que é a qualidade da assistência, e para se prestar assistência de qualidade, é fundamental se adquirir uma noção da existência do processo ou sistematização que fundamenta o serviço que é realizado a cada dia.

\subsubsection{Categoria II: Processo de implantação da VISA}

Nesta categoria os profissionais descrevem como se deu a da Vigilância sanitária em Campos Sales.

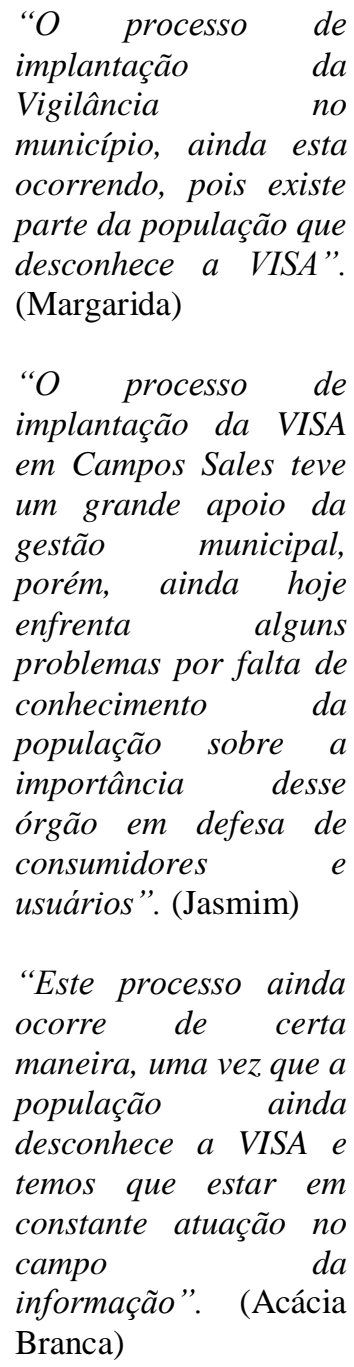

A implementação da Vigilância da Saúde, é um processo complexo que articula o "enfoque populacional" (promoção) com o "enfoque de risco" (proteção) e o enfoque clínico (assistência), constituindo-se de fato uma forma de pensar e de 
agir em saúde, ou seja, uma referência para a formulação de propostas e uma estratégia de organização de um conjunto heterogêneo de políticas e práticas que assumem configurações específicas de acordo com a situação de saúde da populações em cada país, estado ou município (TEIXEIRA e COSTA, 2001).

De acordo com relatos dos profissionais da VISA de Campos Sales, ela ainda está em andamento, isto é, não foi implantado por completo, por se tratar de um órgão complexo, e que abrange vários setores, revelam ainda que o mesmo recebeu o apoio do município. Descrevem que à maioria das pessoas desconhecem a importância da inserção deste setor na cidade, não que isso dificulte na implantação, mas que seria interessante que as pessoas fizessem nota dessa importância e participassem mais dessa fundação.

\subsubsection{Categoria III: Vantagens e desvantagens de implantação do Setor de Vigilância Sanitária.}

Esta categoria, analisada a partir de depoimentos dos participantes da pesquisa, retrata as vantagens e as desvantagens da implantação da Vigilância Sanitária. todo o município e faz bem para a população, talvez a VISA traga um certo desconforto para os comerciantes que não respeitam as leis sanitárias, o que gera uma desvantagem a eles mesmos". (Hortência)

"Vantagens, conseguir conscientizar as pessoas sobre a importância da comercialização de produtos, alimentos $e$ medicamentos bem conservados $e$ desvantagens, para aqueles que não seguem as resoluções, decretos ou leis sanitárias, há sempre um desconforto, pois os mesmos são notificados $e$ muitas vezes encaminhadas para áreas jurídicas". (Jasmim)

As principais dificuldades encontradas ao se iniciar as ações de vigilância sanitária na cidade de Vitória da Conquista - BA. Entre elas pode-se citar: falta de profissionais capacitados em vigilância sanitária; atraso da Secretaria da Saúde do Estado da Bahia em realizar o Curso de Capacitação em Ações Básicas de Vigilância Sanitária; falta de assessoria dos órgãos estaduais para a instalação da vigilância sanitária municipal; desconhecimento pela clientela, da legislação e normas sanitárias; grande número de estabelecimentos sem alvará sanitário (CUNHA e SOLLA, 2001).

Considera-se que a construção de um Sistema de Vigilância em Saúde, na configuração do sistema de saúde brasileiro, como um subsistema do Sistema Único de Saúde, implica um conjunto 
de mudanças na gestão e organização dos serviços de saúde nos âmbito dos três níveis de governo, definindo funções e competências com relação à formulação e implementação de estratégias que visam à implantação e reorganização do processo de planejamento e operacionalização das atividades de Vigilância em Saúde (TEIXEIRA; COSTA, 2002).

Implantar a Vigilância em Saúde é, portanto, um processo complexo que utiliza uma forma de agir e de pensar em saúde que favorece a formulação de propostas e estratégias de organização de políticas e práticas que assumem configurações específicas de acordo com as condições de saúde da população de um território, seja ele país, estado ou município.

Para os profissionais da Vigilância de Campos Sales, as vantagens da implantação desse setor são inúmeras, uma vez que se trata de uma ferramenta tão importante para saúde e que a única desvantagem deste é a falta de compromisso por parte dos comerciantes que não seguem as leis atribuídas pelo órgão, dificultando assim o trabalho dos funcionários da VISA, que precisam fiscalizar tudo isso, além das outras atividades em outros setores que os mesmos executam.

É fundamental para o desenvolvimento e fortalecimento dos serviços de VISA o investimento na formação profissional, na capacitação técnica e na apropriação de novas tecnologias, do contrário as ações de vigilância se podem se transformar num "verdadeiro faz-deconta e da perpetuação de serviços inoperantes e/ou sem efetividade diante do setor regulado, detentor de poder econômico e tecnológico, e das prementes necessidades de saúde da população" (GARIBOTTI; HENNINGTON; SELLI, 2006).

\section{CONSIDERAÇÕES FINAIS}

A Vigilância Sanitária como uma das partes integrantes do Sistema Único de Saúde enfrenta vários desafios que não se restringem apenas às discórdias entre questões mercantis versus saúde, mas a várias demandas externas como a qualidade da formação profissional, a produção e disseminação de conhecimentos e informações atualizadas a pontos conceituais e doutrinários que envolvem todo o arcabouço da área da saúde, e até mesmo as tecnologias que envolvem todo o meio em que se insere a vigilância sanitária (RIBEIRO e JUNGES, 2008).

Nessa pesquisa, procurou-se conhecer e analisar o processo de implantação da Vigilância Sanitária no município de Campos Sales-CE, como também resgatar o conhecimento dos profissionais da VISA sobre a temática em questão.

A investigação teve como base de análise e de estudo o conhecimento dos próprios profissionais da vigilância sanitária, advindo de seu cotidiano, experiência e vivência, pois os sujeitos da pesquisa permitiram que elementos importantes acerca de seus conhecimentos sobre a implantação da VISA fossem descortinados.

Evidenciou-se que existe conhecimento por parte dos profissionais, que os mesmos exercem suas funções voltadas para uma melhor qualidade de vida da população Campossalense, fiscalizando e controlando os riscos para a saúde. Faltando apenas a completa implantação do setor na cidade, mas ressalva que seja um processo lento e complexo, e que, tal população participe e reconheça a importância desse órgão. 


\section{REFERÊNCIAS}

ANDRADE, M. M. Introdução á metodologia do trabalho científico. 6 ed. 2003.

$\begin{array}{lrr}\text { ANVISA. História da Vigilância } & \text { Sanitária no } \\ \text { Brasil. } & \text { Disponível }\end{array}$ http://www.anvisa.gov.br/institucional/historia.h tm Acesso feito em: 07 de janeiro de 2013

COSTA, A. M et al. Cartilha de vigilância sanitária. ANVISA 2 ed. Brasilia. 2002. Disponível em: http://www.anvisa.gov.br/institucional/snvs/coprh/c artilha.pdf Acesso feito em: 07 de janeiro de 2013

COSTA, E. A. Vigilância Sanitária. Caderno de saúde. Belo Horizonte. 2001. Disponível em: http://www.anvisa.gov.br/institucional/snvs/coprh/c ad_saude/cad_saude4.pdf Acesso feito em: 07 de janeiro de 2013

COHEN, M. M; MOURA, M. M. O e TOMAZELLI, J. G Descentralização das ações de Vigilância Sanitária nos municípios em Gestão Plena, Estado do Rio de Janeiro. Rev. bras. epidemiol. vol.7 n.3 São Paulo Sept. 2004

CUNHA, I. C. A e SOLLA, J. J. S. P. Implantação da Vigilância Sanitária em Vitória da Conquista, Bahia. Saúde para Debate, Rio de Janeiro, n. 25, p. 28-36, novembro 2001

DE SETA, M. H.; REIS, L. G. da C.; D, E. V. Gestão da vigilância à saúde. Florianópolis: Departamento de Ciências da Administração/ UFSC; Brasília: CAPES: UAB, 2010. 150p.

GARIBOTTI, V; HENNINGTON, E. A; SELLI, L. A contribuição dos trabalhadores na consolidação dos serviços municipais de vigilância sanitária. Cad. Saúde Pública, v. 22, n. 5, p. 1043-1051, 2006.

GIL, A. C. Como Elaborar Projetos de Pesquisa. Editora Atlas: São Paulo, 2002.

HESSEN, J. Teoria do conhecimento. Martins Fontes. São Paulo. 2000.
LAKATOS, E. M.; MARCONI, M. A. Metodologia científica. 4 ed, São Paulo: Atlas, 2004.

LUCCHESE， G. Globalização e regulação sanitária: os rumos da vigilância sanitária no Brasil. 2001. Tese de Doutorado - Escola Nacional de Saúde Pública, Fundação Oswaldo Cruz, Rio de Janeiro, 2001.

PEREIRA, L. C. B. Reforma Administrativa do Sistema de Saúde. Trabalho apresentado ao Colóquio Técnico prévio à XXV Reunião do Conselho Diretivo do CLAD. Buenos Aires, 25 de outubro, 1995.

PIOVESAN, M. F et al. Vigilância Sanitária: uma proposta de análise dos contextos locais. Rev. bras. epidemiol. vol.8 no. 1 São Paulo Mar. 2005

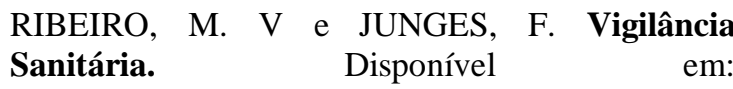
http://www.cpgls.ucg.br/ArquivosUpload/1/File/V MOSTRA DE PRODUO CIENTIFICA/SAUDE/74.pdf . Acesso feito em: 14 de janeiro de 2013

SATO, L. As implicações do conhecimento prático para a vigilância em saúde do trabalhador. Cad. Saúde Públ., Rio de Janeiro, 12(4):489-495, outdez, 1996

TEIXEIRA, C. F e COSTA, E. A. Vigilância da saúde e vigilância sanitária: concepções, estratégias e práticas. 2002 (elaborado para o Programa de Formação dos Agentes locais da Vigilância da Saúde PROFORMAR - FUNASA

TRIVIÑOS，A.N.S. Introdução à pesquisa em ciências sociais. São Paulo: Atlas, 1987. 174p. 